\title{
When it pops-out: Restart all over again from the beginning?
}

\begin{abstract}
An 82-year-old female with a severe aortic stenosis (AS) and a history of acute decompensated heart failure was scheduled for TAVR. A stiff wire accidentally dislodged from the left ventricle before delivering an S3 valve to the aortic valve plane. A successful S3 implantation was achieved by crossing a regular wire using the flex wheel of the delivery system handle to align the nose cone with the aortic orifice followed by an exchange to a stiff wire. This is the very first clinical case report of this technique for a possible bailout procedure during a premature LV guidewire dislodgement, which significantly reduces additional risk, procedural time and cost.
\end{abstract}

\section{Keywords: Transcatheter aortic valve implantation/replacement (TAVI/TAVR) - Complication} Guidewire dislodgment • Bailout

\section{Introduction}

The dislodgment of a stiff wire from the Left ventricular (LV) cavity when a Sapien valve is already in the patient's vasculature is a rare but troublesome complication of transcatheter aortic valve replacement (TAVR). A theoretical bailout technique of retrying to cross the aortic valve with a regular wire using the original system has been suggested in a literature [1]. However, such clinical cases have never been reported.

\section{Case Description}

An 82-year-old female with a severe aortic To the best of our knowledge, this is the very first stenosis (AS) and a history of acute clinical report using this technique as a bailout decompensated heart failure was scheduled for procedure during a premature LV guidewire TAVR. Pre-procedural echocardiography dislodgement, which significantly reduced results indicated the stenosis was very additional risk, procedural time and cost. severe with the measurements of aortic valve area (AVA) 0.28 sq cm, AVA index 0.22 , mean pressure gradient $95.9 \mathrm{mmHg}$ and peak Drs. Yamashita, Iwakiri, Kurebayashi, Suzuki and velocity $6.1 \mathrm{~m} / \mathrm{sec}$. Predilatation was Ohkawa have reported no relationships relevant to performed using a $20-\mathrm{mm}$ balloon with a the contents of this paper to disclose.

\section{Safari XS guidewire (Boston Scientific, Boston, Supplementary Data}

MA, USA) placed in the LV but the guidewire accidentally came out during our struggle to cross a crimped 23-mm Sapien 3 valve (Edwards Lifesciences, Irvine, CA, USA) inside an e-sheath through her to cross the valve orifice with a Radifocus right external iliac artery due to the extreme wire. This guidewire was then successfully delivered friction (Figure 1A). After successfully crossing the iliac artery, the valve was aligned to a proper position and delivered above her native aortic valve over the dislodged wire
Fluoroscopic imaging of the technique. Using its flex function and manual torque of the entire system, the nose cone was manipulated in order into the LV (Video 1) with just the tip of the nose cone going through the valve orifice, which enabled a guidewire exchange to a Safari XS (Video 2).
Takehiro Yamashita1* , Naoki Iwakiri', Eigo Kurebayashi', Masato Suzuki², Yohei Ohkawa²

'Department of Cardiology, Cardiovascular Center, Hokkaido Ohno Memorial Hospital, Sapporo, Hokkaido, Japan

2Department of Cardiovascular Surgery, Cardiovascular Center, Hokkaido Ohno Memorial Hospital, Sapporo, Hokkaido, Japan

*Author for correspondence: Takehiro Yamashita

Email: t_yamashita@cvc-ohno.or.jp Received date: September 13, 2019 Accepted date: September 23, 2019 Published date: September 30, 2019 

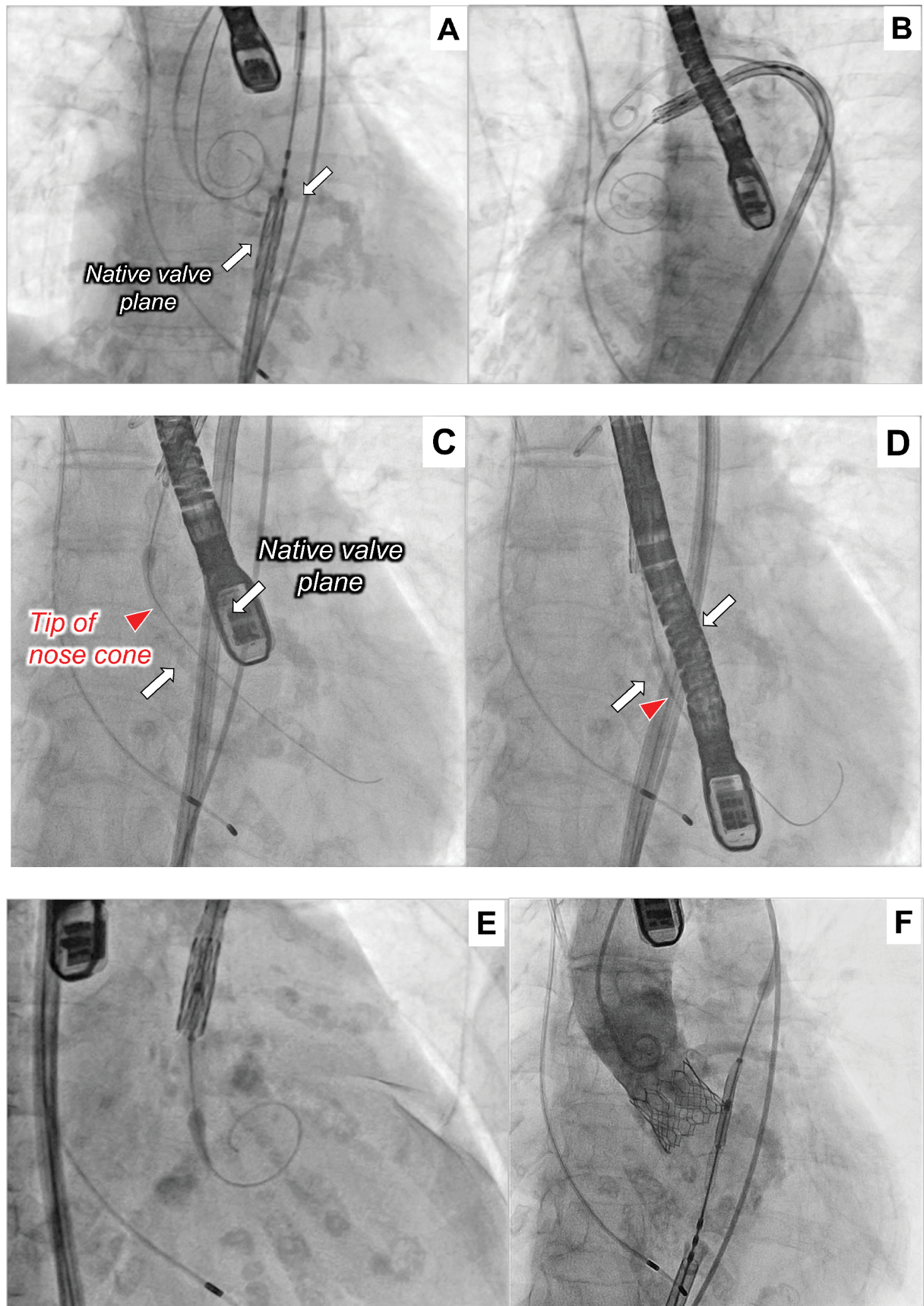

Figure 1: (A) Dislodged Safari XS wire from left ventricular cavity (B) Aligned Sapien 3 valve advancement over dislodged wire above valve orifice (C) Radifocus wire crossing through valve orifice using original system (D) Nose cone crossing through valve plane (E) Guidewire exchange to Safari XS (F) Successful implantation of Sapien 3 valve. 
guidewire was then successfully delivered into the LV (Video 1) with just the tip of the nose cone going through the valve orifice, which enabled a guidewire exchange to a Safari XS (Video 2).

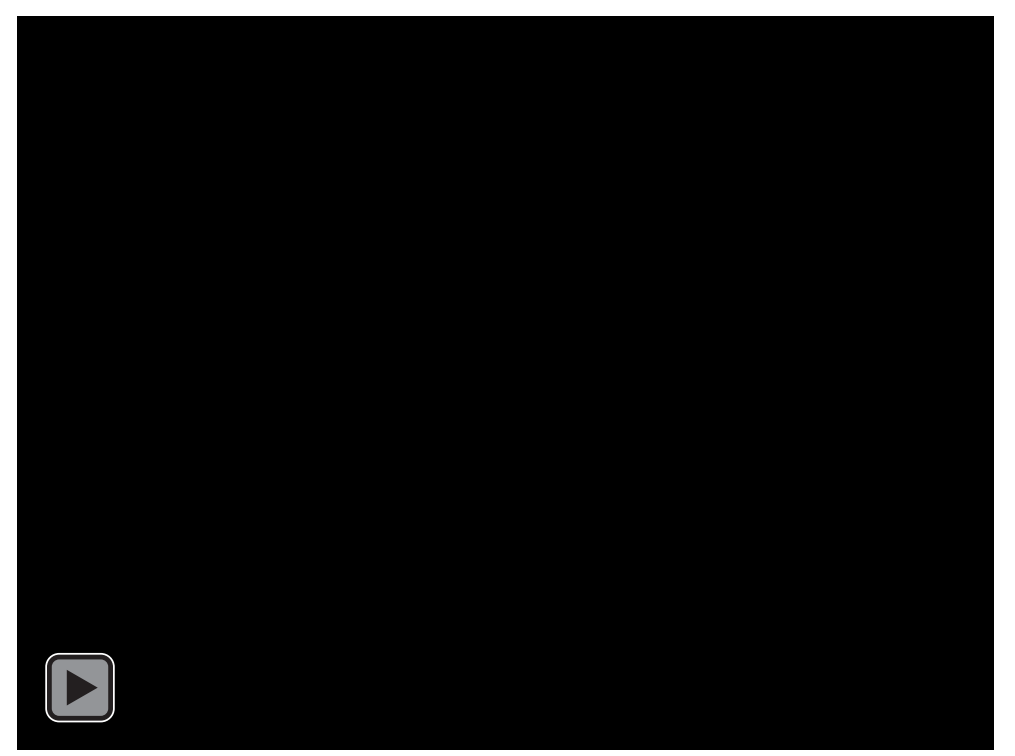

\section{References}

1. Dall'Alla G, Moretti C, Marrozzini C, et al. How should I treat an unexpected deadlock at the time of transcatheter aortic valve prosthesis implantation? Euro Intervention. 13: 256-258 (2017). 